$\underline{\text { Preprint typeset in JHEP style. - HYPER VERSION }}$

\title{
Comments on Noncommutative Sigma Models
}

\author{
Jeff Murugan \\ Department of Mathematics and Applied Mathematics \\ University of Cape Town \\ Private Bag, Rondebosch 7700 \\ South Africa \\ jeff@hbar.mth.uct.ac.za
}

Rory Adams

Department of Physics

University of Cape Town

Private Bag, Rondebosch 7700

South Africa

rory@casimir.phy.uct.ac.za

\begin{abstract}
We review the derivation of a noncommutative version of the nonlinear sigma model on $\mathbb{C P}^{n}$ and it's soliton solutions for finite $\theta$ emphasizing the similarities it bears to the GMS scalar field theory. It is also shown that unlike the scalar theory, some care needs to be taken in defining the topological charge of BPS solitons of the theory due to nonvanishing surface terms in the energy functional. Finally it is shown that, like its commutative analogue, the noncommutative $\mathbb{C P}^{n}$-model also exhibits a non-BPS sector. Unlike the commutative case however, there are some surprises in the noncommutative case that merit further study.
\end{abstract}

Keywords: Noncommutative field theory, Sigma Model solitons, D-Branes. 


\section{Contents}

1. Introduction 1

2. The noncommutative $\mathbb{C P}^{n}$ instanton 3

2.1 Notation 3

2.2 The commutative $\mathbb{C P}^{n}$ sigma model 1

2.3 The noncommutative $\mathbb{C P}^{n}$ sigma model and its BPS solutions 6

3. Non-BPS States 9

3.1 Constructing non-BPS states - The commutative case 9

3.2 Constructing non-BPS states - The noncommutative case 12

3.3 Examples 14

4. $\quad \mathbb{C P}^{n}$ solitons and GMS solitons

5. Conclusions and Discussion 19

\section{Introduction}

Nonlinear sigma models on Kähler and hyper-Kähler target spaces are arguably some of the most important test beds of ideas that invariably find their way into the more daunting arena of physical gauge theories in four dimensional spacetimes. Certainly one of the most favored of such theories is the $d=2$ sigma model with target space $\mathbb{C P}^{n}$ - the $n$-dimensional complex projective space. Like the $d=4$ self-dual YangMills theory it too exhibits asymptotic freedom, conformal invariance and a rich solitonic sector.

A large class of the soliton solutions of the $\mathbb{C P}^{n}$ model are the finite energy lumplike solutions that correspond to holomorphic functions on the two-dimensional base space. The lumps saturate a BPS bound on the $\mathbb{C P}^{n}$ energy functional and are consequently stabilized by some finite topological charge. Although these are by far the most well studied, they are by no means the only solitonic solutions exhibited by the $\mathbb{C P}^{n}$ sigma model. It has been known for some time that certain bound states of such BPS lumps also solve the sigma model equations [7]. These are, however, not solutions of any first order BPS equations and consequently lack the stability properties of the lumps. Nevertheless, there exists a Bäcklund-like solution generating 
technique for generating general (non-BPS) $\mathbb{C P}^{n}$ solutions from a given holomorphic BPS soliton [8, 30]. Yet even in the light of such remarkable similarities between the $\mathbb{C P}^{n}$ sigma model and four-dimensional gauge theories, some differences are quite stark. Chief among these are the lack of a more complete understanding of the soliton moduli space and the absence of a general construction technique like the ADHM method for the $\mathbb{C P}^{n}$ model. One avenue toward a better understanding of the dynamics of the $\mathbb{C P}^{n}$ lumps (as encoded in the moduli space) lies in the deformation of the base space on which the lumps move.

Ever since the realization that the low energy effective theory of D-branes in a $B$ field background [32, 37] is a noncommutative field theory, the deformation of choice has become that of the algebra of smooth functions over the base. This yields a noncommutative $\mathbb{C P}^{n}$ sigma model whose basic solitonic excitations have by now been well documented [26]. In particular, the moduli space metric was explicitly computed for the $1-$ and 2-soliton solutions and shown to be nonsingular and Kähler in both cases [10]. Moreover, in [11] it was shown that, in stark contrast to the commutative case, the noncommutative $\mathbb{C P}^{1}$ sigma model contains a non-BPS sector that is closely tied to the scalar solitons of the GMS field theory [12]. The existence of these new non-BPS excitations of the $\mathbb{C P}^{1}$ model (and, more generally in the $\mathbb{C P} \mathbb{P}^{n}$ model) is certainly intriguing. If nothing else, it is a reminder of the fact that the volume of the solution space of the noncommutative theory is significantly larger than the corresponding commutative one. An interesting question then, is whether the known soliton generating technique of [8, 30] probe this sector of the solution space of the noncommutative $\mathbb{C P}^{n}$ sigma model. As will be demonstrated, this technique is, perhaps surprisingly, deficient in the noncommutative model.

Despite (or perhaps because of) their remarkable simplicity, GMS solitons have had a huge impact on recent literature (see [17] for a recent review). In particular, it was shown in [5, 6] that the algebraic structure of a family of solitonic solutions of the vacuum string field theory equations

$$
\Psi_{m} * \Psi_{m}=\Psi_{m}
$$

is exactly isomorphic to the corresponding one for the GMS solitons of a noncommutative pure scalar field theory. Exploiting this isomorphism leads one to the interpretation of such noncommutative solitons as relics of D23-branes in the low energy limit. If, as in [11] (and later on in this paper), solitonic excitations of the $\mathbb{C P}^{n}$ sigma model exist that can be built up of bound states of scalar solitons, it seems natural to ask whether the noncommutative sigma model solitons may have some interpretation as $D$-brane configurations also. 
The organization of this paper is as follows: After a brief description of the $\mathbb{C P}^{n}$ sigma model and its (commutative) instanton solutions, we proceed to a review of the corresponding noncommutative instantons. While the results in this section are themselves not new, the formulation of the noncommutative sigma model is. By focusing on the formal similarity between the sigma model equations and that of the noncommutative scalar field theory, the BPS bound on the energy functional is rewritten to emphasize the subtlties encountered in defining topological charges of noncommutative objects. This section will establish all the necessary formalism required for the main result of this work: the construction of non-BPS solitons of the noncommutative $\mathbb{C P}^{n}$ sigma model ${ }^{1}$. After the construction of several explicit non-BPS solitons for both the $\mathbb{C P}^{1}$ and $\mathbb{C P}^{2}$ sigma models, the following section is devoted to the comparison of the BPS solitons constructed as holomorphic curves on $\mathbb{C P}^{n}$ and those obtained from bound states of GMS scalar solitons.

\section{The noncommutative $\mathbb{C P}^{n}$ instanton}

By way of establishing notation and some of the conventions, to be followed for the remainder of this paper, we begin by reviewing the construction of the noncommutative soliton solutions of the nonlinear sigma model on a $\mathbb{C P}^{n}$ target space. This section follows closely the recent work of Lee, Lee and Yang 26].

\subsection{Notation}

In studying noncommutative nonlinear sigma models we will, for the most part, be interested in maps $u: \mathbb{R}_{\theta}^{2} \times \mathbb{R} \rightarrow M$ with the target, $M$ a Kähler (or hyper-Kähler) manifold. The sigma model field $u$ takes values in the $\theta$-deformed algebra of functions over $\mathbb{R}_{\theta}^{2}, \mathcal{A}_{\theta}$, whose elements satisfy

$$
f \star g(x)=\left.e^{\frac{i}{2} \theta^{i j} \partial_{i} \partial_{j}^{\prime}} f(x) g\left(x^{\prime}\right)\right|_{x=x^{\prime}}
$$

where $\theta^{i j}=\theta \epsilon^{i j}$ is a nondegenerate, antisymmetric constant matrix and $\theta$ is a positive deformation (noncommutativity) parameter of dimension (mass) ${ }^{2}$. Consequently, coordinates on the noncommutative plane $\mathbb{R}_{\theta}^{2}$ satisfy the Heisenberg algebra $\left[x^{1}, x^{2}\right]:=x^{1} \star x^{2}-x^{2} \star x^{1}=i \theta$. Written in terms of the complex coordinates $z:=\left(x^{1}+i x^{2}\right) / \sqrt{2}$ and $\bar{z}:=\left(x^{1}-i x^{2}\right) / \sqrt{2}$ on $\mathbb{R}_{\theta}^{2}$ the commutator becomes $[z, \bar{z}]=\theta$

\footnotetext{
${ }^{1}$ As this work was nearing completion we became aware of the work of Foda et. al. [9] whose results have significant overlap with our own. The emphasis in [9] is largely on demonstrating that many of the the known results for the construction of general solitonic solutions to the $\mathbb{C P}^{n}$ sigma model are equally applicable in the noncommutative case. The point of our work however, is to highlight the similarities in the description of the BPS and non-BPS solitons of the noncommutative sigma model to that of the scalar GMS solitons hopefully paving the way for further study into the possible embedding of the former into a stringy framework 35
} 
which, up to a rescaling is nothing but the algebra of annihilation and creation operators for the simple harmonic oscillator. By use of the Weyl transform [17], we associate to a function on the noncommutative space an operator acting on an auxiliary Hilbert space $\mathcal{H}=L^{2}(\mathbb{R})$. In a basis of simple harmonic oscillator eigenstates $\mathcal{H}=\bigoplus_{n} \mathbb{C}|n\rangle$. The vacuum $|0\rangle$ is defined, as usual, by the action of the annihilation operator $\widehat{z}$ on it as $\widehat{z}|0\rangle=0$. Further, we have

$$
\begin{aligned}
& \widehat{z}|n\rangle=\sqrt{\theta n}|n-1\rangle, \\
& \widehat{\bar{z}}|n\rangle=\sqrt{\theta(n+1)}|n+1\rangle .
\end{aligned}
$$

This association of functions on the noncommutative space and operators in the Hilbert space is particularly useful in treating differentiation and integration on the noncommutative plane. Under the Weyl map the operations of differentiating and integrating functions over $\mathbb{R}_{\theta}^{2}$ transform to

$$
\begin{aligned}
\partial_{i} & \rightarrow \frac{i}{\theta} \epsilon_{i j}\left[\widehat{x^{j}}, \cdot\right], \\
\int_{\mathbb{R}_{\theta}^{2}} d^{2} x f\left(x^{i}\right) & \rightarrow 2 \pi \theta \operatorname{Tr}_{\mathcal{H}} \widehat{O_{f}}\left(\widehat{x^{i}}\right)=2 \pi \theta \sum_{n \geq 0}\left\langle n\left|\widehat{O_{f}}\right| n\right\rangle .
\end{aligned}
$$

In particular, tracing over the Hilbert space preserves the translational symmetry of the noncommutative plane.

\subsection{The commutative $\mathbb{C P}^{n}$ sigma model}

In this section we collect some well known results on classical nonlinear sigma models on Kähler target spaces [35, 36, 20] that will prove useful in what follows. If $X$ : $\mathbb{R}^{(1,2)} \rightarrow M$ is a map from $(2+1)$-dimensional Minkowski spacetime with standard metric $\eta_{\mu \nu}=\operatorname{diag}(-1,+1,+1)$ to a Kähler target manifold with Riemannian metric $g_{I J}$ then the action for the nonlinear sigma model is

$$
S=\frac{1}{2} \int_{\mathbb{R}^{(1,2)}} d^{3} x \eta^{\mu \nu} \partial_{\mu} X^{I} \partial_{\nu} X^{J} g_{I J} .
$$

The Kähler property of the target manifold means that there exists a covariantly constant real $(1,1)$-tensor field (the almost complex structure) $J$ satisfying $J_{K}^{I} J_{L}^{K}=$ $-\delta_{L}^{I}$ and a closed real two form (the Kähler form) $\Omega=\frac{1}{2} J_{I K} d X^{I} \wedge d X^{K}$. In terms of the almost complex structure and the Kähler form, the energy of a static field configuration may be rearranged to give

$$
E=\frac{1}{4} \int_{\mathbb{R}^{2}} d^{2} x\left(\partial_{i} X^{I} \pm \epsilon_{i}^{j} J_{K}^{I} \partial_{j} X^{K}\right)^{2} \mp \underbrace{\frac{1}{2} \int_{\mathbb{R}^{2}} d^{2} x \Omega_{I K} \epsilon^{l m} \partial_{l} X^{I} \partial_{m} X^{K}}_{2 \pi Q} .
$$

The second term (the topological charge) is just the integral over $\mathbb{R}^{2}$ of the pullback of the Kähler form and is a topological invariant as a result of the fact that $\Omega$ is a 
closed form. This gives the familiar bound on the energy $E \geq 2 \pi|Q|$. The energy bound is saturated by configurations that satisfy the BPS equations

$$
\partial_{i} X^{I} \pm \epsilon_{i}^{j} J_{K}^{I} \partial_{j} X^{K}=0
$$

Since these are the just the Cauchy-Riemann equations, such configurations are nothing but holomorphic curves on the Kähler manifold $M$. Now fix $M$ to be the $n$ dimensional complex projective space $\mathbb{C P}^{n}=\mathbb{C}^{n+1} / \mathbb{C}^{*}$. In terms of the sigma model fields $X^{I}(z, \bar{z}), I=1, \ldots, n$ (the inhomogeneous coordinates on $\mathbb{C P}^{n}$ ) the standard Fubini-Study metric is given by

$$
d s^{2}=4 \frac{\delta_{I J}\left(1+\bar{X}_{K} X_{K}\right)-\bar{X}_{I} X_{J}}{\left(1+\bar{X}_{K} X_{K}\right)^{2}} d X^{I} d \bar{X}^{J} .
$$

The sigma model action is most conveniently formulated in terms of the $\mathbb{C P}^{n}$ homogeneous coordinates $U=\left(u_{1}, \ldots, u_{n+1}\right) \sim\left(\lambda u_{1}, \ldots, \lambda u_{n+1}\right)$ where $\lambda \in \mathbb{C}^{*}$ is a nonzero complex number. Defining $D U:=d U+i U A$, this is given by

$$
S=\int_{\mathbb{R}^{(1,2)}} d^{3} x \eta^{\mu \nu}\left(D_{\mu} U\right)^{\dagger} D_{\nu} U
$$

subject to the constraint $U^{\dagger} U-1=0$. A few points should be immediately apparent from this formulation; the first being the invariance of the action under global $S U(n+$ 1) transformations of the sigma model fields $u_{I} \rightarrow e^{i \alpha} u_{I}$. This is merely a reflection of the equivalence relation defining $\mathbb{C P}^{n}$. The second being the fact that the 'gauge field' is an auxiliary one, completely determined by the sigma model fields $A=i U^{\dagger} d U$. The corresponding equations of motion written in terms of the (homogeneous) sigma model fields are given by

$$
D_{\mu} D^{\mu} U+\left(D_{\mu} U\right)^{\dagger}\left(D^{\mu} U\right) U=0 .
$$

Once again the static energy is bounded by a topological charge $E \geq 2 \pi|Q|$ where now

$$
Q=\frac{i}{2 \pi} \int_{\mathbb{R}^{2}} d^{2} x \epsilon^{i j}\left(D_{i} U\right)^{\dagger}\left(D_{j} U\right)
$$

Reparameterising the sigma model field $U=W / \sqrt{W^{\dagger} W}$ where $W$ is an $(n+1)$ vector, the energy bound is saturated when the first order BPS equations $\partial_{\bar{z}} W=0$ or $\partial_{z} W=0$ are satisfied. These are the instanton and anti-instanton solutions of the $\mathbb{C P}^{n}$ sigma model, constructed by taking $W$ to be a rational function of $z$ and $\bar{z}$ respectively. The topological charge of the soliton is counted as the highest degree of the rational function components of $W$. Before discussing noncommutative generalizations it is worth noting that the $\mathbb{C P}^{n}$ sigma model may be formulated 
completely in terms of the Hermitian projector $P=W\left(W^{\dagger} W\right)^{-1} W^{\dagger}$ in terms of which the action is given by

$$
S=\frac{1}{2} \int_{\mathbb{R}^{(1,2)}} d^{3} x \operatorname{tr} \eta^{\mu \nu}\left(\partial_{\mu} P \partial_{\nu} P\right)
$$

The 'trace' in the integrand is the usual matrix trace operation and the unitary constraint on the sigma model fields $U(z, \bar{z})$ is reflected in $P^{2}=P$. This formulation will prove particularly useful in the construction of non-BPS solitons later.

\subsection{The noncommutative $\mathbb{C P}^{n}$ sigma model and its BPS solutions}

The transition to a noncommutative $\mathbb{C P}^{n}$ sigma model is made, following the standard prescription, by replacing all products occurring in the above formulae with Moyal $\star$-products and subsequently by replacing all noncommutative functions with the associated operators on $\mathcal{H}$. As such, the sigma model action (2.10) becomes

$$
S_{\theta}=\frac{2 \pi}{\theta} \operatorname{Tr}_{\mathcal{H}}\left(\delta_{i j}\left[\widehat{x^{i}}, \widehat{U}^{\dagger}\right](1-P)\left[\widehat{U}, \widehat{x^{j}}\right]\right)
$$

The unitarity condition on the commutative sigma model fields $U^{\dagger} U=1$ becomes an isometry $\widehat{U}^{\dagger} \widehat{U}=1$ on $\mathcal{H}$ (see [17] for more details). In deriving (2.14) use was made of the identity $D_{i} U \rightarrow \frac{i}{\theta} \epsilon_{i j}(1-P)\left[\widehat{x^{j}}, \widehat{U}\right]$ and $P$ is the Hermitian projector as defined above. As in the commutative case, the static action may be rewritten in completely in terms of $P$ as

$$
S_{\theta}=2 \pi \operatorname{Tr}_{\mathcal{H}} \operatorname{tr}\left(\left[P, \widehat{a}^{\dagger}\right][\widehat{a}, P]\right)
$$

after a further rescaling of the coordinates on $\mathbb{R}_{2}^{\theta}$ as $\widehat{z} \rightarrow \sqrt{\theta} \widehat{a}$ and $\widehat{\bar{z}} \rightarrow \sqrt{\theta} \widehat{a}^{\dagger}$. It is worth noting at this juncture that the form of the $\mathbb{C P} \mathbb{P}^{n}$ sigma model action (2.15) is remarkably similar to the kinetic term of the static energy functional of a $(2+1)$ dimensional noncommutative scalar field (eq.(2.2) of ref 13]). Such noncommutative scalar field theories are known to exhibit a spectrum of localized field configurations (GMS solitons) [12, 13] with several interesting properties. Not least among these is the rich structure of the $k$-soliton moduli space; a Kähler de-singularization of $\left(\mathbb{R}^{2}\right)^{k} / S_{k}$, the symmetric product of the single soliton moduli space. It appears though, that this is not unique to the scalar field theory [28, 4]. Indeed a similar resolution of the geometry of the $k$-soliton moduli space, as realized by the noncommutative algebra of projection operators, was demonstrated recently in the noncommutative $\mathbb{C P}^{n}$ sigma model [10] by explicit computation of the Kähler metric on the one- and two-soliton moduli space. In the light of such remarkable evidence, it seems reasonable to ask if there may be further similarities between GMS solitons and those of the $\mathbb{C P}^{n}$ sigma model? With this in mind, it will prove useful to proceed in close analogy with the analysis of GMS solitons. Returning to the $\mathbb{C P}^{n}$ model and 
equating the energy of the configurations with the static action, it is easily seen that, as in the commutative case, the energy is bounded from below. However, as will be demonstrated shortly, some degree of care must be exercised when dealing with higher rank projectors ${ }^{2}$. Naively following 13 it might seem like the energy may be written as

$$
E_{\theta}=2 \pi \operatorname{Tr}_{\mathcal{H}} \operatorname{tr}\left(2 F_{ \pm}(P)^{\dagger} F_{ \pm}(P) \pm P\right) \geq 2 \pi\left|\operatorname{Tr}_{\mathcal{H}} \operatorname{tr} P\right|
$$

where

$$
F_{ \pm}(P)=\left\{\begin{array}{l}
(1-P) \widehat{a} P \\
(1-P) \widehat{a}^{\dagger} P
\end{array}\right.
$$

The inequality would then saturate when the (anti)BPS equations $F_{ \pm}(P)=0$ are satisfied and the topological charge of the BPS solitons takes on a particularly neat expression, being simply the combined matrix and Hilbert space trace of the associated projector. However, this would be too naive! The problem is that $\operatorname{Tr}_{\mathcal{H}} \operatorname{tr}(\mathrm{P})$ is generally infinite and hence cannot represent the soliton charge. Crucial to the resolution of this issue is the understanding that, in the noncommutative case, arguments in the trace may not be permuted with impunity. With this in mind, returning to the static energy (2.15) (and focusing on the BPS case for the moment), it may be seen that ${ }^{3}$

$$
\begin{aligned}
\operatorname{Tr}_{\mathcal{H}} \operatorname{tr}\left[P, \widehat{a}^{\dagger}\right][\widehat{a}, P] & =\operatorname{Tr}_{\mathcal{H}} \operatorname{tr}\left(P \widehat{a}^{\dagger} \widehat{a} P-P \widehat{a}^{\dagger} P \widehat{a}-\widehat{a}^{\dagger} P \widehat{a} P+\widehat{a}^{\dagger} P \widehat{a}\right) \\
& =\operatorname{Tr}_{\mathcal{H}} \operatorname{tr}\left(2 F_{+}(P)^{\dagger} F_{+}(P)+P-\left[\widehat{a}, \widehat{a}^{\dagger} P\right]+\left[P, \widehat{a}^{\dagger} P \widehat{a}\right]\right) .
\end{aligned}
$$

A straightforward computation shows that $\operatorname{Tr}_{\mathcal{H}} \operatorname{tr}\left[P, \widehat{a}^{\dagger} P \widehat{a}\right]=0$ so that the last term may be dropped. Recalling the Weyl prescription mapping functions on a noncommutative space to an auxiliary Hilbert space, the second to last term may be thought of as an "integral of a derivative". As such, this may be evaluated with a noncommutative analogue of Stokes' theorem (see for instance [14, 22])

$$
\operatorname{Tr}_{\mathcal{H}}[\widehat{a}, \mathcal{O}]=\lim _{M \rightarrow \infty} \sqrt{M+1}\langle M+1|\mathcal{O}| M\rangle
$$

where $\mathcal{O}$ is any appropriately well behaved operator on $\mathcal{H}$. For $\mathcal{O}=\operatorname{tr} \widehat{a}^{\dagger} P$, this term is generally nonvanishing and cannot be neglected. With this in mind the energy functional becomes

$$
\begin{aligned}
E_{\theta} & =2 \pi \operatorname{Tr}_{\mathcal{H}} \operatorname{tr}\left(2 F_{+}(P)^{\dagger} F_{+}(P)+P-\left[\widehat{a}, \widehat{a}^{\dagger} P\right]\right) \\
& \geq \underbrace{2 \pi \operatorname{Tr}_{\mathcal{H}} \operatorname{tr}\left(P-\left[\widehat{a}, \widehat{a}^{\dagger} P\right]\right)}_{2 \pi Q_{+}}
\end{aligned}
$$

\footnotetext{
${ }^{2}$ We thank Robert de Mello Koch for drawing our attention to this point.

${ }^{3}$ We would also like to thank O. Lechtenfeld for bringing to our attention ref. 223, 24] in which it was stressed that $E \neq \operatorname{Tr}_{\mathcal{H}} \operatorname{tr} P$ in the more general setting of a noncommutative $U(n)$-valued field in a modified $(2+1)$-dimensional sigma model.
} 
with $F_{+}(P)$ defined as above. Similarly it may be shown that

$$
\begin{aligned}
E_{\theta} & =2 \pi \operatorname{Tr}_{\mathcal{H}} \operatorname{tr}\left(2 F_{-}(P)^{\dagger} F_{-}(P)-\left(P-\left[\widehat{a}, P \widehat{a}^{\dagger}\right]\right)\right) \\
& \geq \underbrace{2 \pi\left|\operatorname{Tr}_{\mathcal{H}} \operatorname{tr}\left(P-\left[\widehat{a}, P \widehat{a}^{\dagger}\right]\right)\right|}_{2 \pi\left|Q_{-}\right|}
\end{aligned}
$$

Again, the energy bound is saturated for configurations for which the (anti)BPS equations $F_{ \pm}(P)=0$ hold. As shown in [26] such solutions are not hard to find; any Hermitian projector constructed from an $(n+1)$-vector $W$ whose components are (anti)holomorphic polynomials will satisfy the above (anti)BPS equations. These are the noncommutative extensions of the instanton solutions of the conventional $\mathbb{C P}^{n}$ sigma model. The static 1 - and 2 -soliton solutions of the noncommutative $\mathbb{C P}^{1}$ model, for example, are given respectively by

$$
W_{1}=\left(\begin{array}{c}
a_{1} \\
\widehat{z}-b_{1}
\end{array}\right), \quad W_{2}=\left(\begin{array}{c}
2 a_{2} \widehat{z}+b_{2} \\
\widehat{z}^{2}+c_{2} \widehat{z}+e_{2}
\end{array}\right) .
$$

The coefficients $a_{1}, \ldots, e_{2} \in \mathbb{C}$ are chosen to coincide with the standard way of writing the corresponding solitons of the commutative theory [10, 36]. These are the complex moduli of the $\mathbb{C P}^{n}$ instantons. The solutions are easily visualized in the small $\theta$ limit by computing the energy density as an operator on the auxiliary Hilbert space and mapping it back to a function on $\mathbb{R}_{\theta}^{2}$ by the Weyl correspondence, i.e. $\widehat{\mathcal{E}} \mapsto \mathcal{E}_{\star}=\mathcal{W}^{-1}(\mathcal{E})$. This is exemplified by the simplest instanton solution [26], $W_{1}=(1, \widehat{z})^{T}$ for which

$$
P=\left(\begin{array}{cc}
\frac{1}{1+\overline{\bar{z}} \widehat{z}} & \frac{1}{1+\overline{\bar{z}} \widehat{\bar{z}}} \\
\widehat{z} \frac{1}{1+\bar{z} \widehat{z}} & \widehat{z} \frac{1}{1+\overline{\bar{z}} \widehat{z}} \widehat{\widehat{z}}
\end{array}\right) .
$$

By way of illustration of the above points it is a useful exercise to compute the topological charge of the above soliton ${ }^{4}$. The trace over $\mathcal{H}$ may be regulated by the introduction of an infrared cutoff $M$ through the restriction to an $M$-dimensional subspace of $\mathcal{H}$ spanned by $\{|0\rangle,|1\rangle, \ldots,|M\rangle\}$ [14. As such

$$
\begin{aligned}
Q_{+} & =\lim _{M \rightarrow \infty} \operatorname{Tr}_{\mathcal{H}_{M}} \operatorname{tr} P-\operatorname{Tr}_{\mathcal{H}_{M}} \operatorname{tr}\left[\widehat{a}, \widehat{a}^{\dagger} P\right] \\
& =\lim _{M \rightarrow \infty} \sum_{n=0}^{M}\left\langle n\left|\frac{1}{1+\widehat{N}}+\frac{1+\widehat{N}}{2+\widehat{N}}\right| n\right\rangle \\
& -\sqrt{M+1}\left\langle M+1\left|\widehat{a}^{\dagger} \frac{1}{1+\widehat{N}}+\frac{\widehat{N}}{1+\widehat{N}} \widehat{a}^{\dagger}\right| M\right\rangle \\
& =\lim _{M \rightarrow \infty} \sum_{n=0}^{M}\left(\frac{1}{1+n}+\frac{1+n}{2+n}\right)-\left(1+\frac{(M+1)^{2}}{M+2}\right)=1
\end{aligned}
$$

\footnotetext{
${ }^{4}$ It is not too difficult to see that the instanton number must be independent of the noncommutativity parameter so in computing $Q_{+}, \theta$ may be set to unity without any loss of generality.
} 
In the small $\theta$ limit, the energy density of the degree one instanton is written in terms of the noncommuting coordinates on the plane as [26]

$$
\mathcal{E}_{\star}=\frac{1}{1+\bar{z} \star z} \star \frac{1}{1+(\bar{z} \star z+\theta)}=\frac{1}{\left(1+\frac{1}{2} r^{2}\right)^{2}}+\mathcal{O}\left(\theta^{2}\right) .
$$

Note that the first corrections to the commutative instanton energy enter only at order $\theta^{2}$ so as long as $\theta$ is small the noncommutative instanton energy density may be adequately approximated by the lowest order term in the $\theta$ perturbation series. A similar computation for the degree two soliton $W_{2}$ (with $a_{2}=c_{2}=0$ ) gives the energy density

$$
\mathcal{E}_{\star}=2 \frac{\left|b_{2}\right|^{2} r^{2}}{\left(\left|b_{2}\right|^{2}+\left|e_{2}\right|^{2}+\frac{1}{4} r^{4}+e_{2} \bar{z}^{2}+\overline{e_{2}} z^{2}\right)^{2}}+\mathcal{O}(\theta) .
$$

These solutions are plotted in fig. 1 for various values of the complex moduli $b_{2}$ and $e_{2}$. It is interesting to note that the low energy scattering of the two degree one instantons is not unlike that of the corresponding configuration of GMS noncommutative scalar solitons [12. Indeed, this scattering property of was explicitly verified in the $\mathbb{C P}^{1}$ case in [10] where the metric on the two-soliton moduli space was directly computed.

\section{Non-BPS States}

In addition to the simplest solutions of the $\mathbb{C P}^{n}$ sigma model, the instanton solutions described above; it is also known that these field theories (and their generalizations to sigma models with Grassmannian target $G r(n, m)=S U(n+m) / S(U(n) \times U(m))$ possess a non-BPS sector consisting essentially of bound states of instantons and anti-instantons [7, 8, 30]. Such solutions solve the (2nd order) equations of motion without saturating any BPS bound on the energy functional and, not protected by supersymmetry, are in general unstable. These classical solutions have also resurfaced recently [20] when it was shown that not only do they solve the $\mathbb{C P}^{n}$ sigma model equations but that they also solve a Dirac-Born-Infeld (DBI) type action pointing to a bulk interpretation of these solitons as D-brane states although the precise states that they correspond to is not yet clear.

\subsection{Constructing non-BPS states - The commutative case}

In this section we aim to continue the analysis carried out in [30] and ask if such nonBPS states persist when the base space of the sigma model is made noncommutative.

To this end, we briefly review the elegant construction employed in [30, modifying it to explicitly treat the $\mathbb{C P}^{n}$ sigma model. The idea behind said approach is rather 

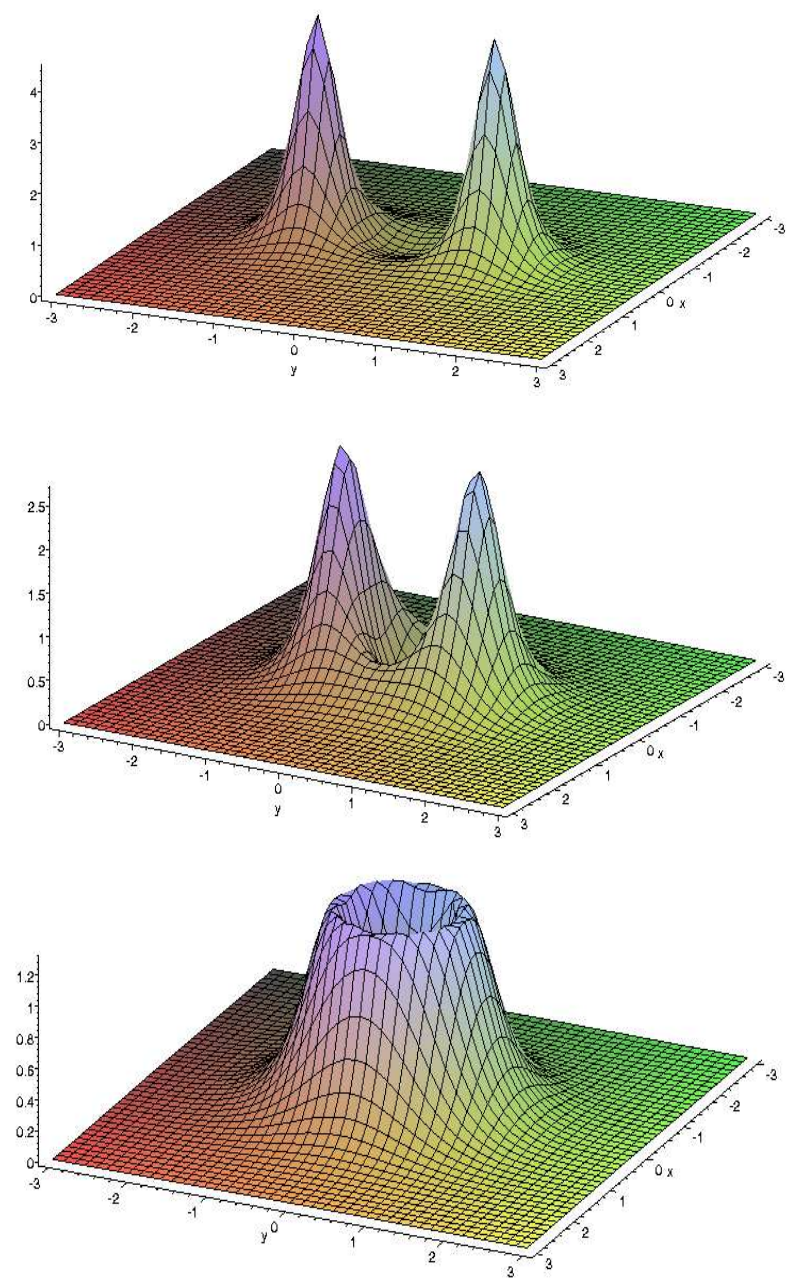

Figure 1: Static 2-soliton solution of the $\mathbb{C P}^{1}$-model with complex moduli $b_{2}=1$, and $e_{2}=1,0.5$ and 0 .

elementary, demanding only a little linear algebra. Given a holomorphic $(n+1)$ vector $\mathbf{f}$ which characterizes the $\mathbb{C P}^{n}$ instanton, a set of $n+1$ vectors $\left\{\mathbf{f}_{1}, \mathbf{f}_{2}, \ldots, \mathbf{f}_{n+1}\right\}$ is constructed from $\mathbf{f}$ (as described below) such that $\operatorname{span}\left\{\mathbf{f}_{1}, \mathbf{f}_{2}, \ldots, \mathbf{f}_{n+1}\right\}=\mathbb{C}^{n+1}$. This set may then be orthonormalized by the conventional Gram-Schmidt procedure and, remarkably, any vector in the resulting orthonormal set is a solution of the $\mathbb{C P}^{n}$ equations of motion. Indeed, this may be seen quite easily as follows; in terms of the complex coordinates $(z, \bar{z})$ on $\mathbb{R}^{2}$ and the Hermitian projector $P$, the $\mathbb{C P}^{n}$ sigma model equations of motion may be written as

$$
\left[\partial_{z} \partial_{\bar{z}} P, P\right]=0
$$

Let $\mathbf{f}$ be some holomorphic $(n+1)$ component vector (any instanton solution will do) and define $\mathbf{f}_{1}:=\mathbf{f}, \mathbf{f}_{2}:=\partial_{z} \mathbf{f}, \ldots, \mathbf{f}_{n+1}:=\partial_{z}^{n} \mathbf{f}$. Assuming linear independence of the $\mathbf{f}_{i}$ for $1 \leq i \leq n+1$ means that they span $\mathbb{C}^{n+1}$. This set may be orthonormalized by 
the usual Gram-Schmidt procedure to give an orthonormal basis for $\mathbb{C}^{n+1}$ as follows: Define $\mathbf{e}_{1}^{\prime}:=\mathbf{f}_{1}, \mathbf{e}_{1}:=\mathbf{e}_{1}^{\prime} /\left(\mathbf{e}_{1}^{\prime \dagger} \cdot \mathbf{e}_{1}^{\prime}\right)^{1 / 2}$ and

$$
\begin{aligned}
& \mathbf{e}_{i}^{\prime}:=\mathbf{f}_{i}-\sum_{j=1}^{i-1} \mathbf{e}_{j}\left(\mathbf{e}_{j}^{\dagger} \cdot \mathbf{f}_{i}\right) \\
& \mathbf{e}_{i}:=\frac{\mathbf{e}_{i}^{\prime}}{\left(\mathbf{e}_{i}^{\prime \dagger} \cdot \mathbf{e}_{i}^{\prime}\right)^{1 / 2}},
\end{aligned}
$$

for $2 \leq i \leq n+1$. It then follows quite straightforwardly that

$$
P_{i}:=\mathbf{e}_{i} \mathbf{e}_{i}^{\dagger} \quad 1 \leq i \leq n+1
$$

is a Hermitian projector. To show that the $\left\{\mathbf{e}_{i}\right\}$ form a set of solutions of the $\mathbb{C P}^{n}$ model, it suffices to show that the $P_{i}$ solve the sigma-model equations of motion (3.1) for any $1 \leq i \leq n+1$. To this end, it will prove useful to define the auxiliary matrix variable

$$
Q_{i}:=\sum_{j=1}^{i-1} \mathbf{e}_{j} \mathbf{e}_{j}^{\dagger}
$$

Clearly $Q$ is also a Hermitian projection operator orthogonal to $P$ since (for fixed $i$ ) $P_{i} Q_{i}=Q_{i} P_{i}=0$. A few lines of algebra together with the identities

$$
\begin{aligned}
\partial_{\bar{z}} \mathbf{e}_{i} & =\sum_{k=1}^{i} \mathbf{e}_{k}\left(\mathbf{e}_{k}^{\dagger} \partial_{\bar{z}} \mathbf{e}_{i}\right) \\
\partial_{z} \mathbf{e}_{i} & =\sum_{k=1}^{i+1} \mathbf{e}_{k}\left(\mathbf{e}_{k}^{\dagger} \partial_{z} \mathbf{e}_{i}\right),
\end{aligned}
$$

establishes that ${ }^{5} \partial_{\bar{z}} Q_{i} Q_{i}=P_{i} \partial_{\bar{z}} Q_{i}=\partial_{\bar{z}}\left(P_{i}+Q_{i}\right)\left(P_{i}+Q_{i}\right)=\partial_{\bar{z}} P_{i} Q_{i}+P_{i} \partial_{\bar{z}} Q_{i}=0$. The last equality of course follows from differentiation of the orthogonality relation satisfied by the $P_{i}$ 's and $Q_{i}$ 's. In much the same way it is also easy to verify that $P_{i} \partial_{z} Q_{i}=\partial_{z} Q_{i}$. Combining these gives

$$
\partial_{\bar{z}} P_{i} P_{i}+\partial_{\bar{z}} Q_{i}=0
$$

and by Hermitian conjugation

$$
P_{i} \partial_{z} P_{i}+\partial_{z} Q_{i}=0
$$

Taking the holomorphic derivative of the former and subtracting the antiholomorphic derivative of the latter gives the desired commutator and completes the proof.

\footnotetext{
${ }^{5}$ For a detailed derivation of these properties of the projection operators we refer the interested reader to [30] and relevant references therein
} 
In the (commutative) classical $\mathbb{C P}^{n}$ sigma model, this procedure can be shown [7, 8] to generate the most general finite action solutions of the sigma model equations of motion. These solutions are interpreted variously as instantons, anti-instantons or unstable noninteracting mixtures thereof. In recent work 20] it was also shown that these non-BPS solitons of the $\mathbb{C P}^{n}$ model are not only finite action solutions of the sigma model but are also finite action solutions of a Dirac-Born-Infeld (DBI) model with a $\mathbb{C P}^{n}$ target space.

\subsection{Constructing non-BPS states - The noncommutative case}

Our focus is however on the noncommutative theory and, as such, one question of interest is whether or not the non-BPS construction above extends to the noncommutative $\mathbb{C P}^{n}$ model. Passing to the noncommutative variables $\widehat{z}$ and $\widehat{\bar{z}}$, results in the equations of motion

$$
[[\widehat{\bar{z}},[\widehat{z}, P]], P]=0
$$

while the BPS and anti-BPS equations are, respectively

$$
\begin{aligned}
& (1-P) \widehat{z} P=0 \\
& (1-P) \widehat{\bar{z}} P=0 .
\end{aligned}
$$

Any solution of the (anti-)BPS equations is also a solution of the Euler-Lagrange equations of motion; a fact that is obvious when the latter is written as $[\widehat{\bar{z}},(1-$ $P) \widehat{z} P]+[\widehat{z}, P \widehat{\bar{z}}(1-P)]=0$ or equivalently $[\widehat{z},(1-P) \widehat{\bar{z}} P]+[\widehat{\bar{z}}, P \widehat{z}(1-P)]=0$. The reverse is, of course certainly not true in general and solutions of (3.8) (if they exist) that do not solve (3.9) are precisely the non-BPS states. We now attempt to find such solutions by adapting the orthonormaization construction of [30]. Let $W$ be a holomorphic $(n+1)$-vector and define

$$
\begin{aligned}
\mathbf{f}_{1} & :=W \\
\mathbf{f}_{2} & :=-\frac{1}{\theta}[\widehat{\bar{z}}, W] \\
& \vdots \\
\mathbf{f}_{k} & :=(-1)^{k-1} \frac{1}{\theta^{k-1}} \underbrace{[\widehat{\bar{z}}, \ldots,[\widehat{\bar{z}}, W] \ldots]}_{(k-1) \text { commutators }} \\
& \vdots
\end{aligned}
$$

The set $\left\{\mathbf{f}_{1}, \mathbf{f}_{2}, \ldots, \mathbf{f}_{n+1}\right\}$ is orthonormalized as follows: Choose $\mathbf{e}_{1}=W\left(1 / \sqrt{W^{\dagger}} W\right)$ and write

$$
\begin{aligned}
\mathbf{e}_{2}^{\prime} & =\mathbf{f}_{2}-\mathbf{e}_{1}\left(\mathbf{e}_{1}^{\dagger} \mathbf{f}_{2}\right)=-\frac{1}{\theta}\left\{[\widehat{\bar{z}}, W]-W \frac{1}{W^{\dagger} W} W^{\dagger}[\widehat{\bar{z}}, W]\right\} \\
& =-\frac{1}{\theta}\left(1-P_{1}\right) \widehat{\bar{z}} W
\end{aligned}
$$


where $P_{1}=W\left(W^{\dagger} W\right)^{-1} W^{\dagger}$ is a Hermitian projection operator and in the last line, use was made of the fact that $W$ is an eigenvector of $P_{1}$ with unit eigenvalue. Computing the norm of $\mathbf{e}^{\prime}{ }_{2}$ as $(1 / \theta) W^{\dagger} \widehat{z}\left(1-P_{1}\right) \widehat{\bar{z}} W$ allows us to write

$$
\mathbf{e}_{2}=-\left(1-P_{1}\right) \widehat{\bar{z}} W \frac{1}{\sqrt{W^{\dagger} \widehat{z}\left(1-P_{1}\right) \hat{z} W}} .
$$

with the associated Hermitian projection operator

$$
P_{2}=\left(1-P_{1}\right) \widehat{\bar{z}} W \frac{1}{W^{\dagger} \widehat{z}\left(1-P_{1}\right) \widehat{\bar{z}} W} W^{\dagger} \widehat{z}\left(1-P_{1}\right)
$$

As in the commutative case, defining $P_{j}:=\mathbf{e}_{j} \mathbf{e}_{j}^{\dagger}$ as the Hermitian projector associated to the $j$ 'th (orthonormal) basis vector we can by iteration construct

$$
\begin{aligned}
\mathbf{e}_{k}^{\prime} & :=(-1)^{k-1} \frac{1}{\theta^{k-1}}\left(1-\sum_{j=1}^{k-1} P_{j}\right)[\widehat{\bar{z}}, \ldots,[\widehat{\bar{z}}, W] \ldots] \\
& =(-1)^{k-1} \frac{1}{\theta^{k-1}}\left(1-\sum_{j=1}^{k-1} P_{j}\right) \widehat{\bar{z}}^{k-1} W
\end{aligned}
$$

where the last equality follows iteratively from the fact that $W \in \operatorname{ker}\left(1-P_{1}\right)$ and $\mathbf{e}_{k}$ is constructed from $\mathbf{e}^{\prime}{ }_{k}$ by the usual normalization. We leave it as a trivial exercise to the reader to verify that the set $\left\{\mathbf{e}_{1}, \ldots, \mathbf{e}_{n+1}\right\}$ is indeed orthonormal. That $\mathbf{e}_{k}$ as constructed above solves the sigma model equations of motion follows in close analogy to the commutative case. For concreteness though, we show this explicitly for the case $k=2$. Observe that the projection operators $P_{1}$ and $P_{2}$ satisfy the relation

$$
\left[\widehat{z}, P_{2}\right] P_{2}+\left[\widehat{z}, P_{1}\right] P_{2}=0
$$

Moreover, the commutative derivative relation $\partial_{z} P_{1}=\mathbf{e}_{2} \mathbf{e}_{2}^{\dagger}\left(\partial_{z} \mathbf{e}_{1}\right) \mathbf{e}_{1}^{\dagger}$ translates in noncommutative coordinates to $\left[\widehat{\bar{z}}, P_{1}\right]=P_{2} \widehat{\bar{z}} P_{1}$ so that $P_{1}$ and $P_{2}$ further satisfy

$$
P_{2}\left[\widehat{\bar{z}}, P_{1}\right]=\left[\widehat{\bar{z}}, P_{1}\right] .
$$

Substituting this into eq.(3.15) and applying the commutator $[\widehat{\bar{z}}, \cdot]$ to the resulting equation gives

$$
\begin{aligned}
{\left[\widehat{\bar{z}},\left[\widehat{z}, P_{2}\right] P_{2}\right] } & +\left[\widehat{\bar{z}},\left[\widehat{z}, P_{1}\right]\right] \\
& =\left[\widehat{z}, P_{2}\right]\left[\widehat{\bar{z}}, P_{2}\right]+\left[\widehat{\bar{z}},\left[\widehat{z}, P_{2}\right]\right] P_{2}+\left[\widehat{\bar{z}},\left[\widehat{z}, P_{1}\right]\right]=0,
\end{aligned}
$$

where in the last step, we have made use of the Jacobi identity $[A,[B, C]]+$ cyclic permutations $=0$ and the Heisenberg algebra satisfied by the noncommuting coordinates. In the latter form it is clear that the first and third terms in (3.17) are 
self-adjoint and so the subtraction from (3.17) of its Hermitian conjugate shows that $P_{2}$ satisfies (3.8) and verifies our claim that $\mathbf{e}_{2}$ is, in fact an exact solution of the noncommutative $\mathbb{C P}^{1}$ sigma model. That this is true, in itself should not be surprising given our construction. A further computation shows that

$$
\left(1-P_{2}\right) \widehat{\bar{z}} P_{2}=\left[\widehat{\bar{z}},\left(P_{1}+P_{2}\right)\right] .
$$

If the commutator on the right hand side of eq.(3.18) vanishes can we conclude that $\mathbf{e}_{2}$ is a non-BPS soliton ${ }^{6}$.

\subsection{Examples}

This noncommutative modification of the Sasaki-Din-Zakrewski (SDZ) construction is perhaps best illustrated by some examples.

- $\underline{\mathbb{C P}^{1}}$ : To begin with, let us consider the case $n=1$. It is a well known fact [7, 8, 38] that for the commutative $\mathbb{C P}^{1}$ sigma model the SDZ construction maps instantons directly to their corresponding anti-instanton solutions. Since the construction yields a complete set of finite action solutions to the sigma model equations of motion it follows then that the commutative $\mathbb{C P}^{1}$ sigma model does not possess a non-BPS spectrum. One might naturally ask if the same is true for the noncommutative $\mathbb{C P}^{1}$ sigma model. It was already shown in [26] that the simplest BPS solution of the noncommutative $\mathbb{C P}^{1}$ model is the $Q=1$ instanton with $W=(1, z)^{T}$ and associated projector (2.23). Substituting this into the expression for $\mathbf{e}_{2}$ in (3.12), simplifying the resulting 2-vector and relabelling the solitonic configuration by $\widetilde{W}_{2}$ we get

$$
\widetilde{W}_{2}=\left(\begin{array}{c}
-\frac{1}{1+\widehat{\bar{z}} \widehat{z}} \widehat{\bar{z}} \\
\frac{1}{1+\widehat{z} \widehat{z}}
\end{array}\right) \sqrt{1+\widehat{\bar{z}} \widehat{z}}=\left(\begin{array}{c}
-\widehat{\bar{z}} \\
1
\end{array}\right) \frac{1}{\sqrt{1+\widehat{\bar{z}} \widehat{z}}} .
$$

This is precisely the normalized anti-holomorphic vector corresponding to the anti-instanton solution expected of the SDZ construction for $\mathbb{C P}^{1}$. This is easily verified by noting that $P_{1}+P_{2}=\mathbb{1}$ so that the commutator on the right hand side of eq.(3.18) vanishes. However, concluding from this that, as in the commutative case, the noncommutative $\mathbb{C P}^{1}$ sigma model does not possess a non-BPS sector would be at best premature (and certainly in this case erroneous). In a remarkable recent work [11] a large class of non-BPS configurations were constructed from meta-stable bound states of solitons and anti-solitons of the GMS noncommutative scalar field theory [12], [13]. The construction of [11] hinges on the fact that in a basis that diagonalizes the

\footnotetext{
${ }^{6}$ This is slightly abusive terminology since (3.18) would ensure only that $\mathbf{e}_{2}$ is not an anti-BPS soliton. We shall take "non-BPS" to mean both equations in (3.9) are nonvanishing.
} 
$(2 \times 2)$ Hermitian projector $P$ associated to a solution of the $\mathbb{C P}^{1}$ sigma model equations, the diagonal entries $\phi_{1}(\widehat{z}, \widehat{\bar{z}})$ and $\phi_{2}(\widehat{z}, \widehat{\bar{z}})$ will also solve (3.8). In particular if $\phi_{1}\left(\phi_{2}\right)$ are taken to be GMS (anti)solitons satisfying $\left(1-\phi_{1}\right) \widehat{z} \phi_{1}=0$ and $\left(1-\phi_{2}\right) \widehat{\bar{z}} \phi_{2}=0$ respectively, then $P$ does not solve either of the equations in (3.9) and the corresponding field configuration $W$ is a non-BPS soliton of the $\mathbb{C P}^{1}$ sigma model. From this example it is alarmingly clear that the SDZ construction does not saturate the set of solutions of the noncommutative $\mathbb{C P}^{1}$ sigma model.

- $\underline{\mathbb{C P}^{2}}$ : Having shown that the modified SDZ construction is insensitive to the non-BPS spectrum of the noncommutative $\mathbb{C P}^{1}$ sigma model we now consider the $n=2$ case. Analysis of these solutions will prove useful in facilitating comparison with the work of $\left[11\right.$. The simplest instanton solution of the $\mathbb{C P}^{2}$ sigma model is $W=\left(1, \widehat{z}, \widehat{z}^{2}\right)^{T}$. The corresponding Hermitian projector is computed to be

$$
P_{1}:=W \frac{1}{W^{\dagger} W} W^{\dagger}=\left(\begin{array}{ccc}
\frac{1}{A} & \frac{1}{A} \widehat{\bar{z}} & \frac{1}{A} \widehat{\bar{z}}^{2} \\
\widehat{z} \frac{1}{A} & \widehat{z} \frac{1}{A} \widehat{\bar{z}} & \widehat{z} \frac{1}{A} \widehat{\bar{z}}^{2} \\
\widehat{z}^{2} \frac{1}{A} & \widehat{z}^{2} \frac{1}{A} \widehat{\bar{z}} & \widehat{z}^{2} \frac{1}{A} \widehat{\bar{z}}^{2}
\end{array}\right)
$$

where $A(\widehat{\bar{z}} \widehat{z})=1+\hat{\bar{z}} \widehat{z}-\theta \hat{\bar{z}} \widehat{z}+(\hat{\bar{z}} \widehat{z})^{2}$ is the square modulus of $W$. Using the relations $\widehat{\bar{z}} f(\widehat{\bar{z}} \widehat{z})=f(\widehat{\bar{z}} \widehat{z}-\theta) \widehat{\bar{z}}$ and $\widehat{z} f(\widehat{\bar{z}} \widehat{z})=f(\widehat{\bar{z}} \widehat{z}+\theta) \widehat{z}$ we find

$$
\begin{aligned}
\widetilde{W}_{2} & :=\mathbf{e}_{2}=-\left(1-P_{1}\right) \widehat{\bar{z}} W \frac{1}{\sqrt{W^{\dagger} \widehat{z}\left(1-P_{1}\right) \hat{\bar{z}} W}} \\
& =\left(\begin{array}{c}
-\widehat{\bar{z}}(1+2 \widehat{\bar{z}} \widehat{z}) \\
1-\theta \hat{\bar{z}} \widehat{z}-(\widehat{\bar{z}} \widehat{z})^{2} \\
\widehat{z}(\widehat{z} \widehat{\bar{z}}+2)
\end{array}\right) \frac{1}{\sqrt{B(\widehat{\bar{z}} \widehat{z})}},
\end{aligned}
$$

with $B(\widehat{\bar{z}} \widehat{z})=1+\theta+\left(5+6 \theta+\theta^{2}\right) \widehat{\bar{z}} \widehat{z}+\left(6+6 \theta+\theta^{2}\right)(\widehat{\bar{z}} \widehat{z})^{2}+(5+2 \theta)(\hat{\bar{z}} \widehat{z})^{3}+(\widehat{\bar{z}} \widehat{z})^{4}$. It is straightforward (but tedious) to compute $P_{2}=\widetilde{W}_{2}^{\dagger} \widetilde{W}_{2}$ and check that the commutator $\left[\widehat{\bar{z}},\left(P_{1}+P_{2}\right)\right]$ is nonvanishing and so conclude that $\widetilde{W_{2}}$ is a genuine non-BPS soliton of the $\mathbb{C P}^{2}$ sigma model. As a check we find that in the $\theta \rightarrow 0$ limit $\widetilde{W_{2}}$ becomes

$$
\left(\begin{array}{c}
-\bar{z}\left(1+r^{2}\right) \\
1-\frac{1}{4} r^{4} \\
z\left(\frac{1}{2} r^{2}+2\right)
\end{array}\right) \frac{1}{\sqrt{1+\frac{5}{2} r^{2}+\frac{3}{2} r^{4}+\frac{5}{8} r^{6}+\frac{1}{16} r^{8}}}
$$

in complete agreement with [7].

- $\underline{\mathbb{C P}^{2}}$ : As a final illustration of the construction technique we start with the $\mathbb{C P}^{2}$ instanton $W=\left(\widehat{z}^{2}+1, \widehat{z}^{2}-1,2 \widehat{z}\right)^{T}$. With $A(\widehat{\bar{z}} \widehat{z})=2+(4-2 \theta) \widehat{\bar{z}} \widehat{z}+2(\widehat{\bar{z}} \widehat{z})^{2}$, the 
corresponding projection operator is written

$$
P_{1}=\left(\begin{array}{ccc}
\left(\widehat{z}^{2}+1\right) \frac{1}{A}\left(\widehat{\bar{z}}^{2}+1\right) & \left(\widehat{z}^{2}+1\right) \frac{1}{A}\left(\widehat{\bar{z}}^{2}-1\right) & 2\left(\widehat{z}^{2}+1\right) \frac{1}{A} \widehat{\bar{z}} \\
\left(\widehat{z}^{2}-1\right) \frac{1}{A}\left(\widehat{\bar{z}}^{2}+1\right) & \left(\widehat{z}^{2}-1\right) \frac{1}{A}\left(\widehat{\bar{z}}^{2}-1\right) & 2\left(\widehat{z}^{2}-1\right) \frac{1}{A} \widehat{\bar{z}} \\
2 \widehat{z} \frac{1}{A}\left(\widehat{\bar{z}}^{2}+1\right) & 2 \widehat{z} \frac{1}{A}\left(\widehat{\bar{z}}^{2}-1\right) & 4 z \frac{1}{A} \widehat{\bar{z}}
\end{array}\right) .
$$

If we define $B(\hat{\bar{z}} \widehat{z})=1+2 \theta+\left(4+6 \theta+2 \theta^{2}\right) \widehat{\bar{z}} \widehat{z}+\left(6+6 \theta+\theta^{2}\right)(\widehat{\bar{z}} \widehat{z})^{2}+(4+$ $2 \theta)(\bar{z} z)^{3}+(\widehat{\bar{z}} \widehat{z})^{4}$ then the (normalized) non-BPS soliton constructed from $W$ may be written

$$
\widetilde{W_{2}}=\left[\left(\begin{array}{c}
\widehat{z}-\widehat{\bar{z}} \\
\widehat{z}+\widehat{\bar{z}} \\
1-\widehat{\bar{z}} \widehat{z}
\end{array}\right)(1+\widehat{\bar{z}} \widehat{z})+\theta\left(\begin{array}{c}
\widehat{z} \\
\widehat{z} \\
-\widehat{z} \widehat{\bar{z}}
\end{array}\right)\right] \frac{1}{\sqrt{B(\widehat{\bar{z}} \widehat{z})}} .
$$

In this form the commutative limit is very conveniently investigated. Sending $\theta$ to zero and noticing that $B(\widehat{\bar{z}} \widehat{z}) \rightarrow(1+\bar{z} z)^{4}$ reduces $\widetilde{W_{2}}$ to

$$
\left(\begin{array}{c}
z-\bar{z} \\
z+\bar{z} \\
1-\frac{1}{2} r^{2}
\end{array}\right) \frac{1}{1+\frac{1}{2} r^{2}}
$$

Again, this corresponds exactly to what is expected in the commutative limit. As can be seen from the soliton energy density (see Fig.2) the non-BPS state is formed from a bound state of two degree- $1 \mathbb{C P}^{2}$ instantons and two antiinstantons all coincident at the origin.

\section{4. $\mathbb{C P}^{n}$ solitons and GMS solitons}

The existence of a non-BPS spectrum of the noncommutative $\mathbb{C P}^{1}$ model is intruging [11]; even more so since the contruction of non-BPS solitonic confugurations is so intimately connected to the noncommutative scalar solitons of [12]. It seems only natural then to try and probe this connection further in the hope of a deeper understanding of the space of solutions to the noncommutative $\mathbb{C P}^{n}$ sigma model. Returning to the BPS solitons of Sec.(2.3), it may be immediately seen that an alternative construction of degree $k$ solitons would be to take the Hermitian projector $P$ in the diagonal representation (as in [1])

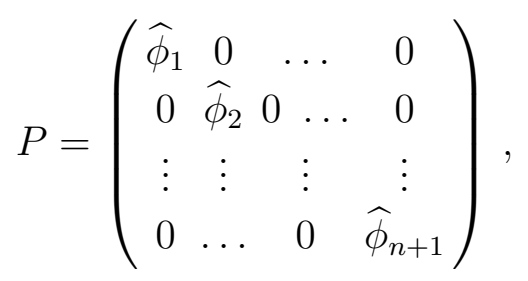

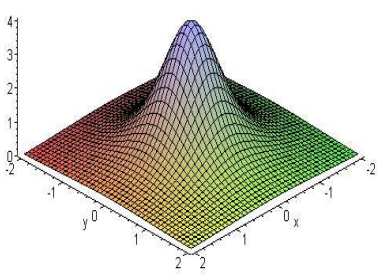

Figure 2: $\mathrm{A} \mathbb{C P}^{2}$ bound state consisting of two instantons and two antiinstantons all coincident at the origin with total energy density four times that of a single instanton. 
where each of the $\widehat{\phi}^{\prime}$ s satisfy ${ }^{7}$

$$
\left(1-\widehat{\phi}_{i}\right) \widehat{a} \widehat{\phi}_{i}=0
$$

and $\sum_{i} \operatorname{Rank}\left(\widehat{\phi}_{i}\right)=k$. This necessarily implies that $(1-P) \widehat{a} P=0$ - precisely the condition that $P$ corresponds to a BPS $\mathbb{C P}^{n}$ configuration. In general, solutions to (4.2) are parameterized by $k_{i}$ complex coherent state vectors $\left|z_{i}^{a}\right\rangle:=\exp \left(z_{i}^{a} \widehat{a}^{\dagger}\right)|0\rangle$ with

$$
\widehat{\phi}_{i}=\sum_{a, b=1}^{k_{i}}\left|z_{i}^{a}\right\rangle \frac{1}{\left\langle z_{i}^{a} \mid z_{i}^{b}\right\rangle}\left\langle z_{i}^{b}\right|
$$

These are, of course, the noncommutative scalar solitons of the GMS model 12, 13. Apparently then, in addition to the standard construction of $k$-soliton solutions of the noncommutative $\mathbb{C P}^{n}$ sigma model [26, 10, 11], such solutions may also be constructed from stacking GMS solitons of appropriate charge on the plane. The emergence of a non-BPS sector of the $\mathbb{C P}^{1}$ model for finite $\theta$ via such a construction lends much weight in favour of this claim. It will be shown in this section however that, at least for (anti-)BPS solutions, such an 'alternative' construction should have been expected since (4.1) is just the diagonal represention of the usual Hermitian projector associated to BPS solutions of the $\mathbb{C P}^{n}$ sigma model. This is most easily illustrated for the case of the static 1 -soliton solution of the noncommutative $\mathbb{C P}^{1}$ model for which $P$ is given by

$$
P=\left(\begin{array}{cc}
\frac{1}{1+\widehat{N}} & \frac{1}{1+\widehat{N}} \widehat{a}^{\dagger} \\
\widehat{a} \frac{1}{1+\widehat{N}} & \frac{1+\widehat{N}}{2+\widehat{N}}
\end{array}\right)
$$

after setting $\theta=1$. Denoting $|I\rangle=(1,0)^{T}$ and $|I I\rangle=(0,1)^{T}$, an eigenvector of $P$ with eigenvalue $\lambda$ may be expanded as

$$
|\Psi\rangle=\left|\psi_{1}\right\rangle \otimes|I\rangle+\left|\psi_{2}\right\rangle \otimes|I\rangle
$$

where $\left|\psi_{i}\right\rangle \in \mathcal{H}$ may be expanded in the harmonic oscillator basis as $\left|\psi_{i}\right\rangle=\sum_{n=0}^{\infty} c_{n, i}|n\rangle$. The action of $P$ on the basis elements is easily determined to be

$$
\begin{aligned}
& P|n\rangle \otimes|I\rangle=\frac{1}{1+n}|n\rangle \otimes|I\rangle+\frac{\sqrt{n}}{1+n}|n-1\rangle \otimes|I I\rangle \\
& P|n\rangle \otimes|I\rangle=\frac{\sqrt{1+n}}{2+n}|n+1\rangle \otimes|I\rangle+\frac{1+n}{2+n}|n\rangle \otimes|I I\rangle
\end{aligned}
$$

\footnotetext{
${ }^{7}$ For concreteness, we shall focus only on the BPS solutions with the understanding that similar relations hold for the anti-BPS case.
} 
so that in terms of the expansion coeffients $c_{n, 1}$ and $c_{n, 2}$ the eigenvalue equation for $P$ becomes

$$
\begin{aligned}
& \sum_{n=0}^{\infty}\left(\frac{c_{n, 1}-\lambda(n+1) c_{n, 1}+\sqrt{n} c_{n-1,2}}{n+1}\right)|n\rangle \otimes|I\rangle \\
+ & \sum_{n=0}^{\infty}\left(\frac{c_{n+1,1} \sqrt{n+1}+(n+1) c_{n, 2}-\lambda(n+2) c_{n, 2}}{n+2}\right)|n\rangle \otimes|I\rangle=0 .
\end{aligned}
$$

Since $P$ is a projection operator, $\lambda=0$ or 1 . Choosing first $\lambda=1$ reduces (4.7) to

$$
c_{n, 2}=\sqrt{n+1} c_{n+1,1}
$$

which fixes completely the $c_{n, 2}$ coefficients in terms of the $c_{n, 1}$ 's and gives

$$
\begin{aligned}
\left|\psi_{1}\right\rangle & =\sum_{n=0}^{\infty} c_{n, 1}|n\rangle \\
\left|\psi_{2}\right\rangle & =\sum_{n=0}^{\infty} \sqrt{n+1} c_{n+1,1}|n\rangle
\end{aligned}
$$

In an orthonormal eigenbasis $\left\{\left|\chi_{1}\right\rangle,\left|\chi_{2}\right\rangle\right\}$ the diagonal representation of the $2 \times 2$ matrix $P$ is

$$
P=\left|\chi_{1}\right\rangle\left\langle\chi_{1}|\otimes| I\right\rangle\left\langle I|+| \chi_{2}\right\rangle\left\langle\chi_{2}|\otimes| I I\right\rangle\langle I I|
$$

It remains only to fix the $c_{n, 1}$ coefficients. This may be done by noting that $P$ is a solution of the $\mathbb{C P}^{n}$ BPS equations only if $\left(1-\left|\chi_{i}\right\rangle\left\langle\chi_{i}\right|\right) \widehat{a}\left|\chi_{i}\right\rangle\left\langle\chi_{i}\right|=0$ i.e., if $\left|\chi_{i}\right\rangle$ is an eigenstate of $\widehat{a}$. Written in terms of the expansion coefficients, this condition reads

$$
c_{n, 1}=\frac{\chi_{1}^{n}}{\sqrt{n !}} c_{0,1}
$$

where $\chi_{1}$ is the eigenvalue corresponding to $\left|\chi_{1}\right\rangle$. This is, of course, expected of a coherent state in a harmonic oscillator basis. It may also quite easily be established that the $\lambda=0$ case is trivial, yeilding $\left|\chi_{1}\right\rangle=\left|\chi_{2}\right\rangle=0$. A straightforward application of Gram-Schmidt orthonormalization finds

$$
\left|\chi_{1}\right\rangle=\sum_{n=0}^{\infty} \frac{c_{n, 1}}{\sqrt{\sum_{m \geq 0}\left|c_{m, 1}\right|^{2}}}|n\rangle, \quad\left|\chi_{2}\right\rangle=0
$$

leaving only the first term in (4.10). Without loss of generality, the residual degree of freedom in (4.11) may be fixed by choosing $c_{0,1}=1$. The eigenvalues $\chi_{1}$ are then interpreted as the complex location moduli of the solitons. For example, the simplest choice of $\chi_{1}=0$ produces a degree 1 soliton localized at the origin,

$$
P=\left(\begin{array}{cc}
|0\rangle\langle 0| & 0 \\
0 & 0
\end{array}\right)
$$


The end result then is that in a diagonal representation the $\mathbb{C P}^{1} 1$-soliton solution is nothing but a unit rank GMS soliton. These results are easily extended to show that the $k$-soliton solution of the $\mathbb{C P}^{n}$ sigma model in a diagonalizing basis may be written in the form (4.1). The interpretation here is that any degree $k \mathbb{C P}^{n}$ soliton may be built up of appropriate rank GMS solitons. Note, however, that the diagonalization is non unitary - given a rank $k$ matrix of the form (4.1) it is not possible in general to associate to it a unique (non-diagonal) Hermitian projection matrix that is also a solution of the sigma model equations. The set of solutions to the sigma model equations that are of the form (4.1) is considerably larger that those formed by adapted commutative constructions. As such, it is not surprising that the solution space of the noncommutative $\mathbb{C P}^{n}$ sigma model is much larger than the corresponding commutative theory. In particular, as shown in [11, certain quasistable configurations of GMS solitons and anti-solitons form non-BPS states of of the noncommutative $\mathbb{C P}^{n}$ sigma model that have zero size in the vanishing $\theta$ limit. Such solutions cannot be realized as the diagonalization of any non-diagonal solution of the sigma model equations.

\section{Conclusions and Discussion}

In trying to understand the connection (if any) between solitonic excitations of the noncommutative sigma model on $\mathbb{C P}^{n}$ and $D$-brane configurations in string theory, we have reformulated the noncommutative $\mathbb{C P}^{n}$ model of [26] in a way that makes manifest the similarities (and differences) with the GMS scalar field theory. In doing so it becomes evident that the BPS solitons of the sigma model are no more immune from problems in the definition of the toplogical charge than any of the higher codimension solitons of, say, four-dimensional noncommutative gauge theory [21]. In this case, the naive calculation of the topological charge is in fact incorrect and must be supplemented by the addition of a nonvanishing "surface term" of the form $\operatorname{Tr}_{\mathcal{H}} \operatorname{tr}[\widehat{a}, \cdot]$. Such terms vanish for GMS solitons and are consequently dropped in that case.

We have also extended the SDZ construction for non-BPS solitons of the $\mathbb{C P}^{n}$ model from known holomorphic (BPS) lumps and constructed explicit solutions for the case of $\mathbb{C P}^{1}$ and $\mathbb{C P}^{2}$. Unlike the commutative case though, the noncommutative SDZ construction does not yield the most general solitonic solutions of the sigma model equations. This incompleteness is due largely to the emergence of a new length scale in the problem as set by the noncommutativity parameter $\theta$. Evidently, as shown in [11], the solution space of the noncommutative sigma model is significantly larger than the corresponding commutative one with the additional (non-BPB) solitons made up of quasi-stable bound states of GMS solitons. While this construction

might seem independent of the standard one, we have shown that, at least for the 
case of BPS solitons, they arise in the diagonalization of the Hermitian projector associated to a given BPS soliton.

The (commutative) $\mathbb{C P}^{n}$ model is well known to arise as the low energy (infinite coupling) limit of a gauged linear sigma model with Fayet-Illiopolous D-term [31]. One class of solitonic excitations of this model are the votex solutions of the first order BPS equations

$$
\begin{aligned}
F_{12}+e^{2}\left(\phi^{\dagger} \phi-1\right) & =0 \\
D_{\bar{z}} \phi & =0 \\
\Phi=\int d^{2} x F_{12} & =2 \pi k>0
\end{aligned}
$$

In the $e^{2} \rightarrow \infty$ limit these vortex solitons decend to the usual $\mathbb{C P}^{n}$ lumps (when $\phi$ is an $(n+1)$-component complex vector). It was recently demonstrated that the noncommutative version of the linear sigma model in question also exhibits vortex excitations that are solutions of the noncommutative vortex equations [2, 3, 22, 34]

$$
\begin{aligned}
1+\left[C^{\dagger}, C\right] & =\gamma\left(\phi^{\dagger} \phi-1\right) \\
\phi a+C \phi & =0 \\
\operatorname{Tr}_{\mathcal{H}}\left(1-\left[C^{\dagger}, C\right]\right) & =-k
\end{aligned}
$$

where $\gamma=\theta e^{2}$ is a dimensionless parameter and $C$ is effectively the (noncommutative) Abelian gauge field. The exact vortex solutions of [2, 22, 34] manifest in the $\gamma \rightarrow \infty$ limit (in which the vortex equations become tractable). Usually this limit is taken by sending $\theta \rightarrow \infty$ but clearly may also arise in the infinite coupling limit; the vortex equations are insensitive to which. As such, it is not unreasonable to expect that the exact vortex solutions decend to the lump solutions of the noncommutative $\mathbb{C P}^{n}$ sigma model. Moreover, the noncommutative Abelian Higgs model may be embedded in a $(5+1)$-dimensional, $\mathcal{N}=1$ supersymmetric theory so that the vortices of the former become BPS 3-branes which preserve half of the supersymmetries. So, more than just another academic exercise, the study of the infinite coupling limit of the noncommutative Abelian Higgs model may provide valuable insight into a string theoretic interpretation of the noncommutative $\mathbb{C P}^{n}$ lumps. These issues will be addressed in future work.

Yet another intriguing avenue for a stringy interpretation of the BPS solitonic excitations of the $\mathbb{C P}^{n}$ sigma model is that offered by the work of [25]. Drawing on the (tree level) equivalence of $N=2$ open string theory and self-dual Yang-Mills theory in $(2+2)$-dimensions [29] it was argued that the effective field theory induced by open $N=2$ strings in a Kähler $B$-field background on the worldvolume of $n$ coincident $D 2$-branes is a modified $U(n)$ sigma model. The latter was also shown to 
exhibit solitonic solutions which were elegantly constructed using a 'dressing method' [23, 24]. From this perspective, a string theoretic interpretation already exists: an $m$-soliton solution to the noncommutative $\mathbb{C P}^{n}$-sigma model should correspond to $m$ D0-branes inside $(n+1)$ coincident $D 2$-branes. A positive identification of the solitonic excitations of the sigma model with the $D 0-D 2$ system would, however, require more than just a matching of the energies of the two systems; it remains to compute the fluctuation spectra around the respective configurations. This is certainly an exciting avenue and warrants further research ${ }^{8}$.

\section{Acknowledgments}

J.M. acknowledges support by the Sainsbury-Lindbury Trust and a research associateship of the University of Cape Town. R.A is supported by a University of Cape Town research associateship. We would like to thank Philip Candelas, George Ellis, Bogdan Florea, Robert de Mello Koch, Joao Roderigues, David Tong and especially Amanda Weltman for useful discussions and valuable insights. J.M. is especially grateful to Philip Candelas, for his kind hospitality at the Mathematical Institute at Oxford University and to ISCAP (Columbia University) for kind hospitality during the time this work was being completed.

\section{References}

[1] M.F. Atiyah and N.J. Hitchin Phys. Lett A 107, 21 (1985)

[2] D. Bak "Exact Multivortex Solutions in Noncommutative Abelian Higgs Theory" Phys. Lett. B 495, 251-255, (2000), hep-th/0008204

[3] D. Bak, K. Lee and J-H. Park "Noncommutative Vortex Solitons" Phys. Rev. D 63, 125010 (2001), hep-th/0011099

[4] H.W. Barden and N.A. Nekrasov "Instantons, Hilbert Schemes and Integrability." (2001), hep-th/0103204

[5] L. Bonora, D. Mamone and M. Salizzoni "Vacuum String Field Theory with a B-field" JHEP 0204, 020 (2002), hep-th/0203188

[6] L. Bonora, D. Mamone and M. Salizzoni "Vacuum String Field Theory ancestors of the GMS solitons" hep-th/0207044

[7] A.M. Din and W.J. Zakrzewski Nucl. Phys. B 174, 397 (1980)

[8] A.M. Din and W.J. Zakrzewski Nucl. Phys. B 237, 461 (1984)

\footnotetext{
${ }^{8}$ We thank O. Lechtenfeld for several useful comments in this regard.
} 
[9] O. Foda, I. Jack and D.R.T. Jones "General classical solutions in the noncommutative $\mathbb{C P}^{n-1}$ model,"(2001), hep-th/0209111

[10] K. Futura, T. Inami, H. Nakjima and M Yamamoto "Low-energy dynamics of noncommutative $\mathbb{C P}^{1}$ solitons in $2+1$ dynamics," (2002), hep-th/02030125

[11] K. Futura, T. Inami, H. Nakjima and M Yamamoto "Non-BPS Solutions of the Noncommutative $\mathbb{C P}^{1}$ Model in (2+1) Dimensions," (2002), hep-th/02030125

[12] R. Gopakumar , S. Minwalla and A. Strominger "Noncommutative Solitons" JHEP 0005, 020 (2000), hep-th/0003160

[13] R. Gopakumar, M. Headrick and M. Spradlin "On Noncommutative Multisolitons." (2001), hep-th/0103256

[14] D.J. Gross and N.A. Nekrasov JHEP 0103, 044 (2001), hep-th/0010090

[15] J. Gutowski and G. Papadopoulos "The moduli spaces of worldvolume solitons," Phys. Lett. B 432, 98 (1998) hep-th/9802186

[16] J. Gutowski "The moduli space of $\mathbb{C P}^{1}$ stinrgy cosmic lumps," Phys. Lett. B 525, $150(2002)$

[17] J.A. Harvey, "Komaba Lectures on Noncommutative Solitons and D-Branes," (2001), hep-th/0102076

[18] J.A. Harvey, P. Kraus, F. Larsen and E. J. Martinec "D-Branes and Strings as Noncommutative solitons," JHEP 0007, 042 (2000), hep-th/0005031

[19] J.A. Harvey, P. Kraus and F. Larsen "Exact Noncommutative Solitons," JHEP 0012, 024 (2000), hep-th/0010060

[20] T. Ioannidou, G. Papadopoulos and P.M. Sutcliffe "Non-BPS Dirac-Born-Infeld Solitons" JHEP 9909, 016 (1999), hep-th/9907156

[21] T. Ishikawa, S-I. Kuroki. and S Akifumi "Instanton Number Calculus On Noncommutative $\mathbb{R}^{4}$." JHEP 0208, 028 (2002), hep-th/0201196

[22] D. Jatka, G. Mandal and S. Wadia "Nielsen-Olesen Vortices in Noncommutative Abelian Higgs Model' JHEP 0009, 018 (2000), hep-th/0007078

[23] O.Lechtenfeld and A.D.Popov "Noncommutative Multi-solitons in 2+1 dimensions" JHEP 0111, 040 (2001), hep-th/0106213

[24] O.Lechtenfeld and A.D.Popov "Scattering of Noncommutative Solitons in $2+1$ dimensions" Phys. Lett. B 523, 178-184 (2001), hep-th/0108118

[25] O.Lechtenfeld, A.D.Popov and B. Spendig "Noncommutative Solitons in Open N=2 String Theory" JHEP 0106, 011 (2001), hep-th/0103196 
[26] B-H. Lee, K. Lee and H.S. Yang Phys. Lett B 498, 277-284 (2001), hep-th/0007140

[27] N.S. Manton Phys. Lett B 110, 54 (1982)

[28] N.A. Nekrasov "Noncommutative instantons revisited." (2000), hep-th/0010017

[29] H. Ooguri and C. Vafa "Geometry of N=2 Strings" Nucl. Phys. B 361, 469-518 (1991)

[30] R. Sasaki J. Math. Phys. 26, 7 (1985)

[31] B.J. Schroers "The Spectrum of Bogomol'nyi solitons in gauged linear sigma models" Nucl. Phys. B 475, 440-468 (1996), hep-th/0210010

[32] N. Seiberg and E. Witten "String Theory and Noncommutative Geometry" JHEP 09, 032 (1999), hep-th/9908142

[33] J.M. Speight "The deformed conifold as a geometry on the space of unit charge $\mathbb{C P}^{1}$ lumps," Phys. Lett. B 511, 295 (2001)

[34] D. Tong "The Moduli Space of Noncommutative Vortices" hep-th/0210010

[35] P. Townsend "Brane Theory Solitons: Cargese Lectures 1999," (2000), hep-th/0004039

[36] R.S. Ward Phys. Lett B 158, 424 (1985)

[37] E. Witten "Noncommutative Geometry and String Field Theory" Nucl. Phys. B 268, 253 (1986)

[38] W.J. Zakrzewski Low Dimensional Sigma Models, IOP (1989) 